\title{
FUNDAMENTAL STUDY ON ULTRASOUND SENSING TECHNOLOGY USING PARAMETRIC SOUND
}

\author{
HIDEHARU TAKAHASHI, NARUKI SHOJI, ATSUSHI ITO \& HIROSHIGE KIKURA \\ Tokyo Institute of Technology, Japan
}

\begin{abstract}
At the Fukushima Daiichi Nuclear Power Plant (NPP) in Japan, investigations of NPP conditions are conducted before retrieval of fuel debris to achieve the decommissioning of NPP. In this study, we focused on the ultrasound measurement technique for the investigation, since ultrasound techniques can overcome environmental issues such as a cloudy, dark and highly radiation dose environment. However, ultrasound is strongly attenuated in the air even if the distance is short. We attempted to apply parametric sound source technology, which is expected to make the measurable distance longer for this problem. Parametric sound source is characterized by higher directivity and lower attenuation. Therefore, it is expected that shape measurement using a parametric sound source can measure long distances. In this paper, as the fundamental study on ultrasound sensing for measurement application inside of a nuclear reactor using this technique, we investigated the measurable length and conducted shape measurement for the simplified shape sample of the simulated fuel debris. In addition, we tried to measure the sample under mist environment, which is difficult to visualize using optical measurements such as camera. Moreover, we developed the small ultrasonic measurement device for carrying by robot transportation system.
\end{abstract}

Keywords: ultrasound, parametric sound, shape measurement, remote measurement, robot, Fukushima-Daiichi NPP, visualization, sensor, decommissioning, pulser-receiver.

\section{INTRODUCTION}

Regarding the decommissioning of TEPCO's Fukushima Daiichi Nuclear Power Plant (NPP) in Japan, various technical issues remain [1]. One of the major issues is understanding the conditions inside the reactor. There are photographic images from a robot that swims underwater or on a rail from the penetration, instead of a robot that runs on the grating, but basically the robot goes around the grating and measures the surrounding area. On the other hand, there is little information on the relevant part and upper part of the core where fuel should have actually existed. Therefore, Tokyo Institute of Technology in Japan has been conducting various technical research on the decommissioning. One of them is advanced super dragon articulated robot arm (Fig. 1) in Tokyo Institute of Technology, Japan [2], [3]. It is a long-articulated arm with a total length of $10 \mathrm{~m}$ that can be freely operated in up, down, left and right directions by driving it with a lightweight, high-strength radiation-resistant wire. The arm including the measurement devise can be made to enter the reactor.

In this study, we focused on the ultrasound measurement technique for the investigation using the robot arm, since ultrasound technique can overcome environmental issues such as a cloudy, dark and highly radiation dose environment. However, ultrasound is strongly attenuated in the air even if the distance is short. We attempted to apply parametric sound source technology which is expected to make the measurable distance longer for this problem. Parametric sound source is characterized by higher directivity, lower attenuation. Therefore, it is expected that shape measurement using parametric sound source can measure long distance. In this paper, as the fundamental study on ultrasound sensing for measurement application inside of nuclear reactor using this technique, we investigated the measurable length and conducted shape measurement for the simplified shape sample of the simulated fuel debris. In addition, we tried to measure the sample under mist environment, which 


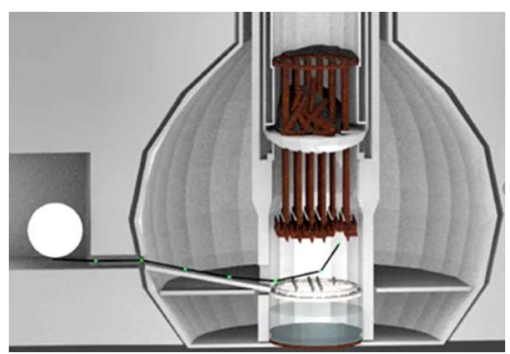

Figure 1: Challenge to investigation of fuel debris in reactor pressure vessel by an advanced super dragon articulated robot arm.

is difficult to visualize using optical measurements such as camera. Moreover, we are developing small ultrasonic measurement devices to be loaded on the robot and developed new portable ultrasonic measurement hardware devices to suit our measurement techniques. In this paper, finally, we described them.

\section{EXPERIMENTAL APPARATUS AND METHOD}

\subsection{Measurable length experiment using parametric sound}

In this paper, a parametric sound is a sound generated from a modulation of high-frequency carrier ultrasonic wave. A measurable length experiment was carried out to verify the distance that can be measured. Fig. 2 shows a schematic diagram of the experimental apparatus. The experimental apparatus consisted by transmission and reception units using a pulser (RITEC, RPR-4000), a transmitter (Japan Probe, 0.4 K14 $\times 20$ N AR1342), a receiver (SPL Ltd., UT1007-Z325R), an external amplifier, a receiver (Japan Probe, JPR-600C), A/D converter (National Instruments, USB-5133) and a control PC. The transmitting and receiving transducers were facing each other as shown in the figure, and ultrasonic pulses were transmitted and received using a normal ultrasound $(400 \mathrm{kHz})$ and parametric sound at a measurement distance $\mathrm{L}$ from 0 to $3,500 \mathrm{~mm}$ between the transducers. Parametric ultrasound $50 \mathrm{kHz}$ was used with $400 \mathrm{kHz}$ ultrasonic carrier wave.

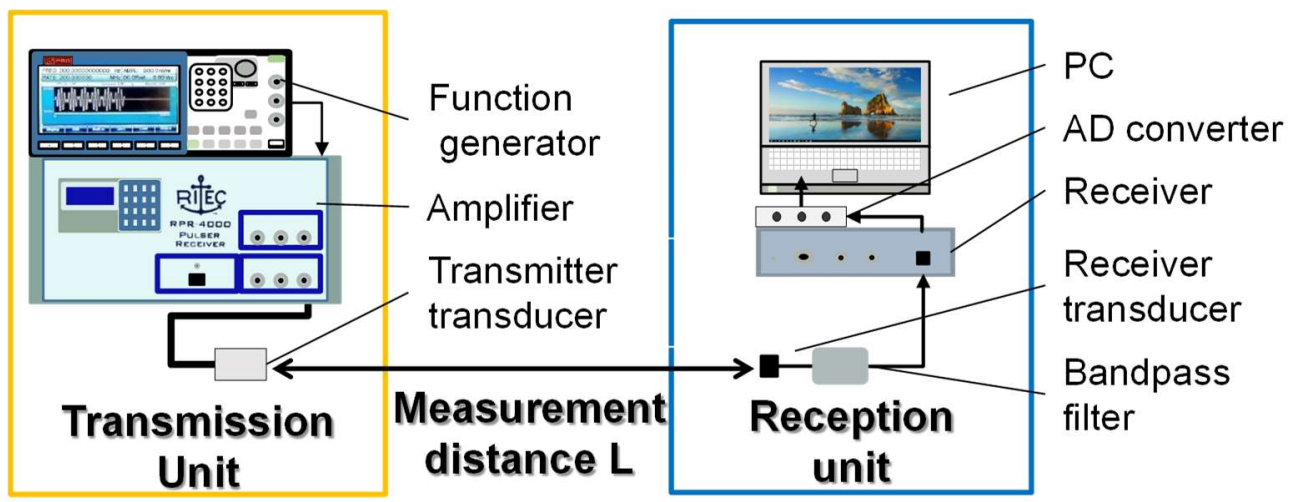

Figure 2: Schematic diagram of experimental apparatus. 


\subsection{Shape measurement experiment using parametric sound}

Fig. 3 shows an overview of the experimental system. The experimental apparatus consisted of an aluminum frame XYZ stage, a pulser (RITEC RPR-4000), a transmitter transducer (Japan Probe, $0.4 \mathrm{~K} 14 \times 20 \mathrm{~N}$ AR1342), a receiver transducer (Nippon Ceramic, PR40-18N), an external amplifier, and a receiver (Japan Probe, JPR-600), an A/D converter (National Instruments, USB-5133), a control PC, and a simplified test sample (simulated fuel debris). A cylinder with a diameter of $50 \mathrm{~mm}$ and a height of $50 \mathrm{~mm}$ was used as the test sample. An image of the sample is shown in Fig. 4. For the measurement, the distances from the transmitter transducer to the bottom of the test sample were $250 \mathrm{~mm}$ and $500 \mathrm{~mm}$. The pitch of the sensor movement was set to $\mathrm{d}=3 \mathrm{~mm}$. For the detection of the ultrasonic received echo signal, if the minimum signal-to-noise ratio of the received echo signal is greater than $6 \mathrm{~dB}$, it is considered to be reproducible, and the propagation time where the absolute value of the received signal strength is maximum is set as the received position of the reflected wave signal. The sample height ( $\mathrm{Z}$ direction) at each position was calculated from Time Of Flight (TOF) method and the shape was reconstructed by each height information at each XY direction positions.

(1) Function Generator

(2) Pulser/ Receiver for Transmitting

(3) Transducer (Transmitter)

(4) Transducer (Receiver)

(5) XYZ Stage

(6) Stage Controller

(7) External Amplifier

(8) Pulser/ Receiver for Receiving

(9) A/D Converter

(10) $\mathrm{PC}$

(11) Simplified Sample (Simulated fuel debris)

(12) Mist Generator

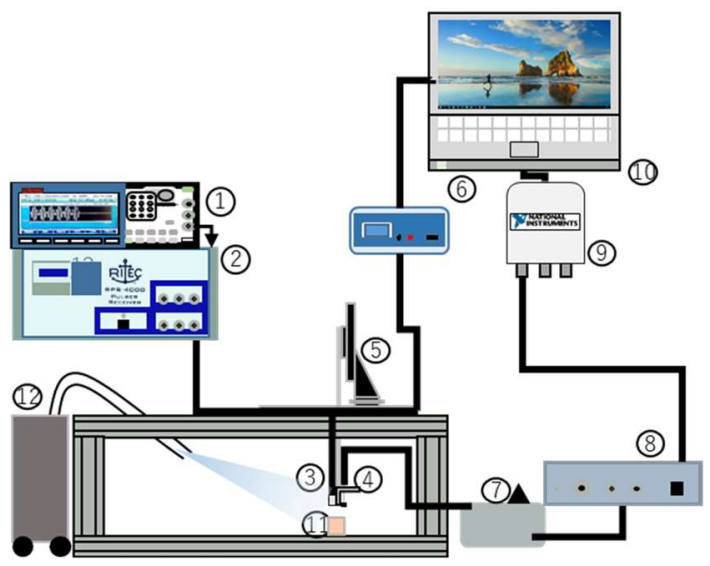

Figure 3: Schematic diagram of experimental apparatus.
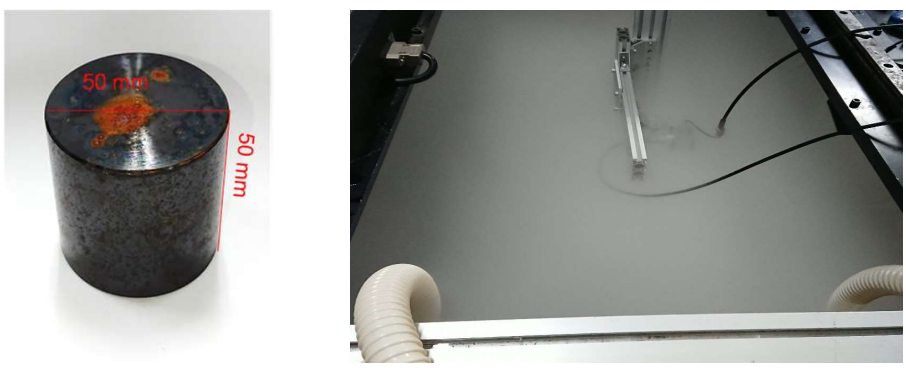

Figure 4: Photograph of simplified shape sample of the simulated fuel debris and the experimental environment under mist condition. 
The proposed measurement method uses ultrasonic propagating in the air, and it is concerned that the ultrasonic waves may be affected by mist in the air, which attenuates and scatters the ultrasonic waves. Therefore, we tried to measure the sample without or with mist environment condition (Fig. 4), which is difficult to visualize using optical measurements such as camera.
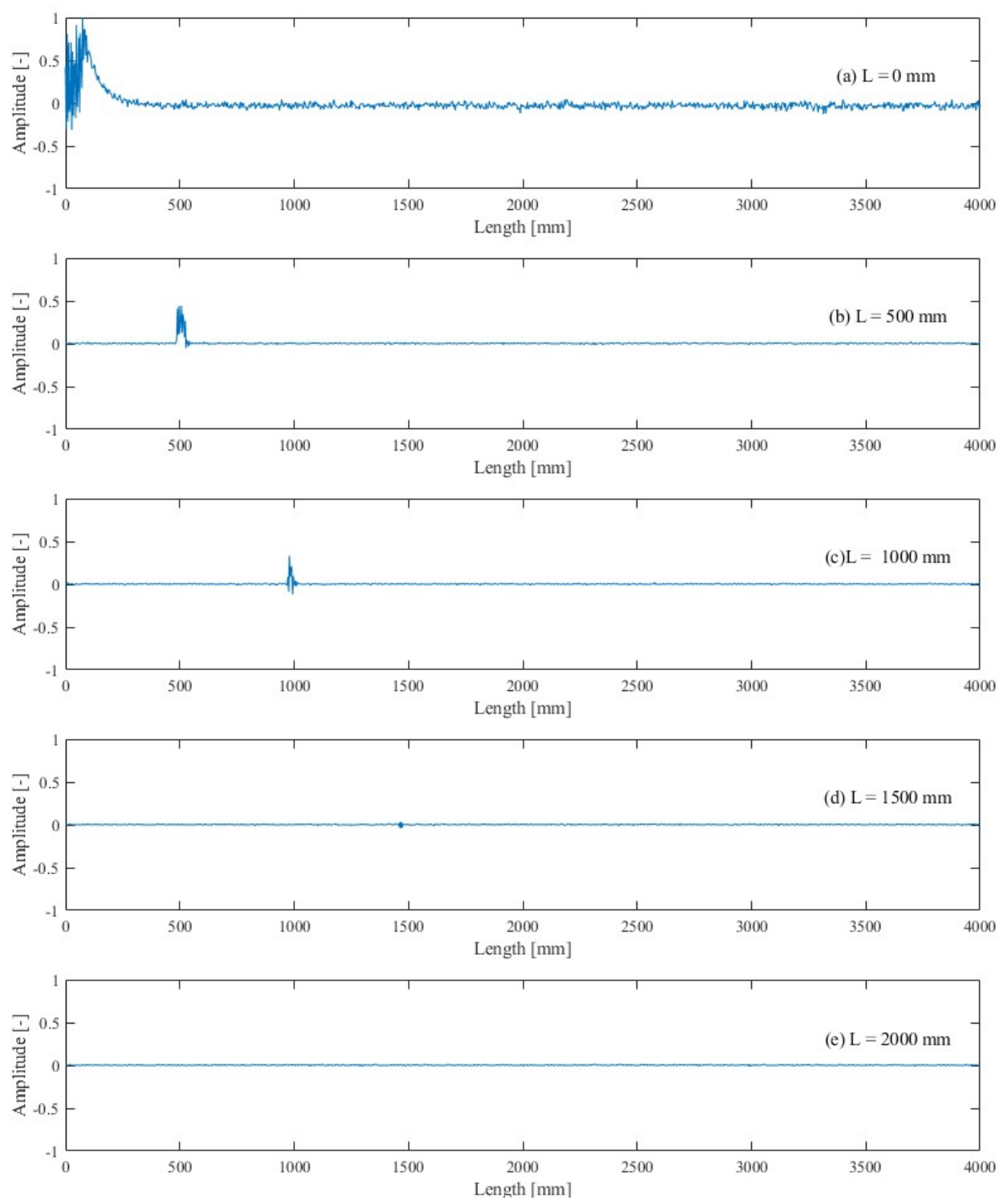

Figure 5: Measurable length measurement results using normal $400 \mathrm{kHz}$ ultrasound. 


\section{EXPERIMENTAL RESULTS AND DISCUSION}

\subsection{Measurable length experiment using parametric sound}

Figs 5 and 6 show the results of measurable length experiment with normal ultrasound $(400 \mathrm{kHz})$ and with a parametric sound. The vertical axis represents the signal strength, and is normalized so that the maximum value of the signal at each measurement is 1 . The horizontal axis shows the distance calculated from the time elapsed since the pulse was transmitted. Figure shows the received signal intensity for the measurement distance.
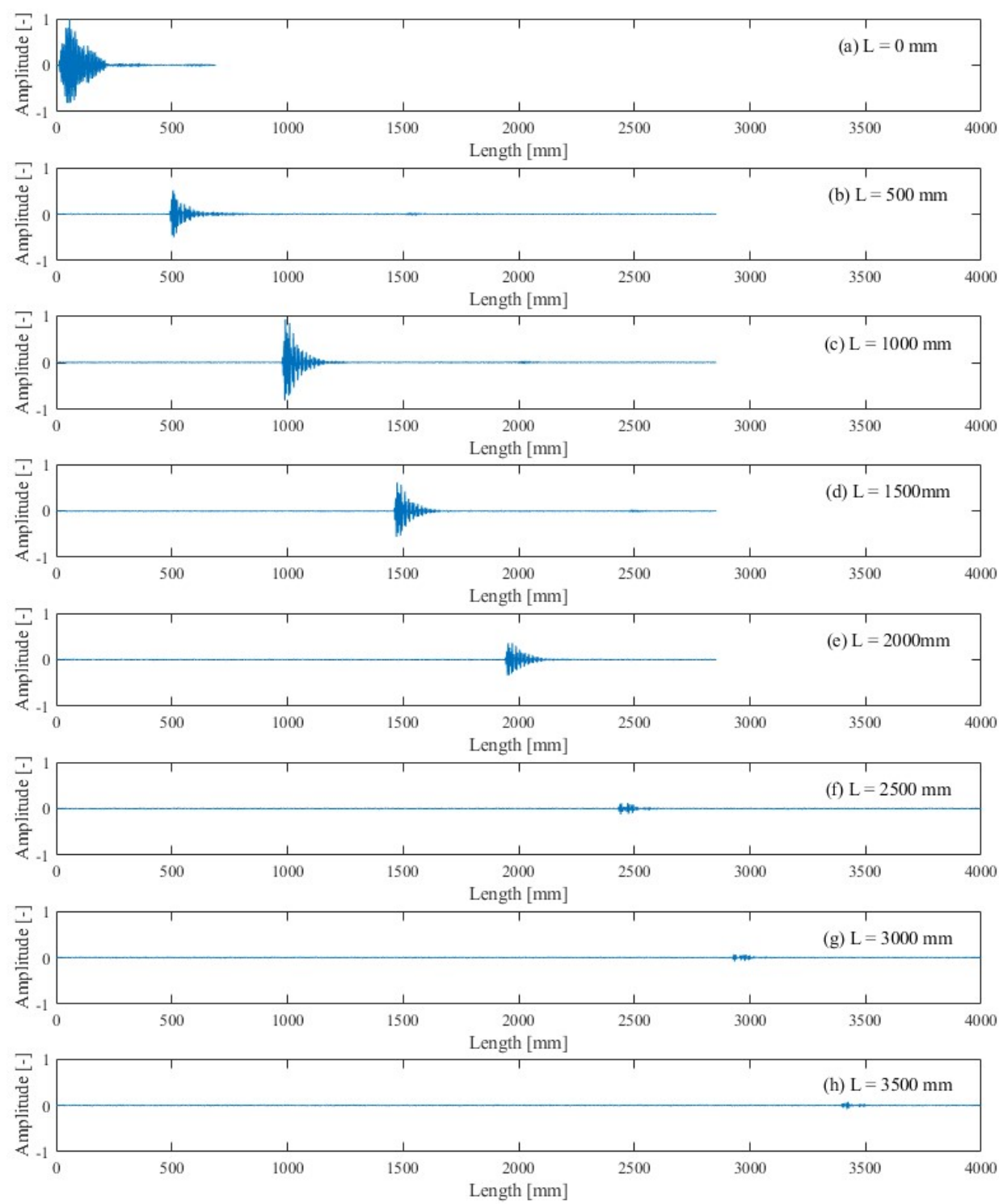

Figure 6: Measurable length measurement results using parametric sound. 
Fig. 7 shows comparison of measurable signal distance. As shown in Fig. 7, the parametric sound was able to transmit and receive data at a distance of 3,500 $\mathrm{mm}$, which is the maximum distance that can be measured in this experiment. On the other hand, it was difficult to measure at 2,000 mm, when we use normal $400 \mathrm{kHz}$ ultrasound. The use of a parametric sound is an effective way to extend the range of ultrasonic measurements.

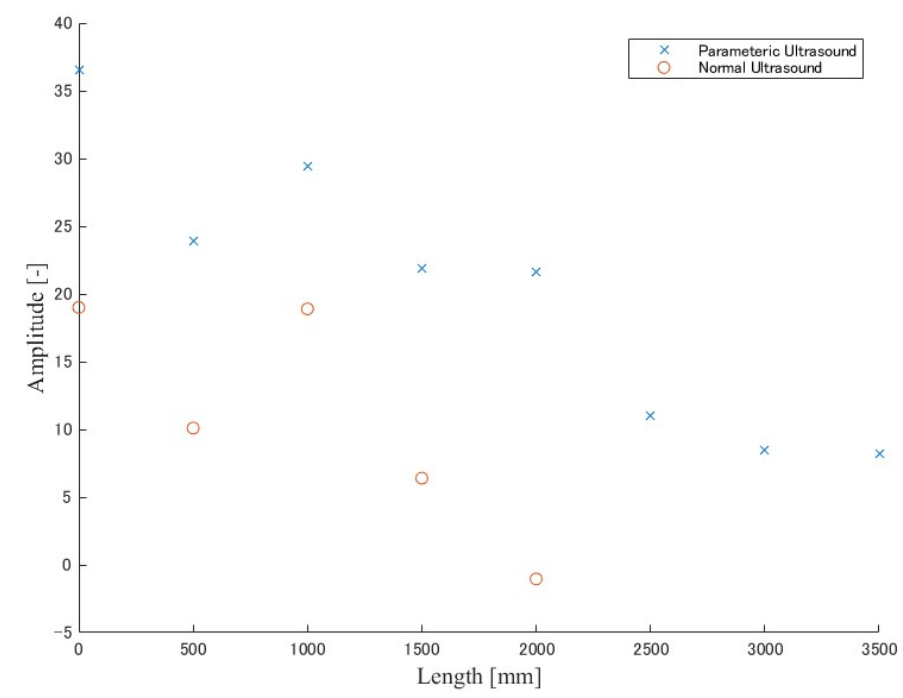

Figure 7: Comparison of measurable reflection signal distance.

\subsection{Shape measurement experiment using parametric sound}

Fig. 8 shows the reconstructed shape of the sample at distance of $250 \mathrm{~mm}$ to the specimen's bottom surface in no mist condition. From this figure, we can see that the reconstruction of the outline of the shape is well done. Since the error of the cylinder height was $3.3 \%$ on average, the measurements in the direction of ultrasound transmission ( $\mathrm{Z}$ axis direction) were accurate. On the other hand, in the radial direction, the error in the evaluation of the circular equivalent was $-20 \%$.

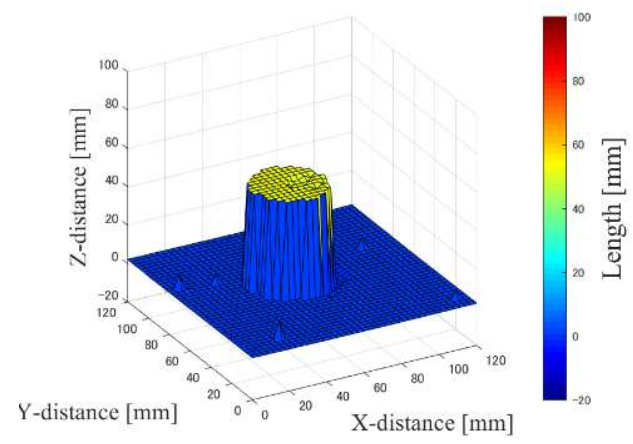

Figure 8: Shape measurement result using parametric sound (Measurement distance $=$ $250 \mathrm{~mm}$, without mist condition). 
Fig. 9 shows the reconstructed shape of a sample at distance of $250 \mathrm{~mm}$ from the bottom of the sample in the presence of mist. This figure shows that the reconstruction of the outline can be achieved even in the presence of mist. The error in the height of the cylinder was $3.2 \%$ on average, so that the measurements in the direction of ultrasound transmission were accurate. On the other hand, in the radial direction, the error in the circular equivalent diameter evaluation was $-20 \%$, which is considered to be due to the small size of the sample in relation to the width of the ultrasonic beam.

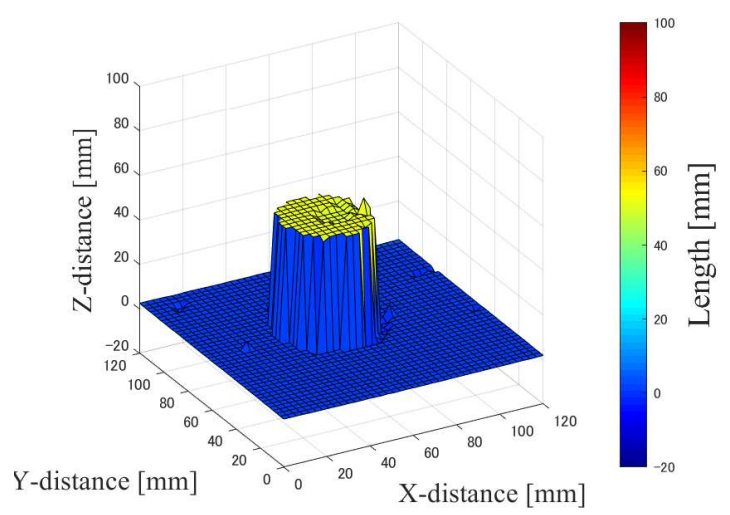

Figure 9: Shape measurement result using parametric sound (Measurement distance $=$ $250 \mathrm{~mm}$, with mist condition).

Fig. 10 shows the reconstructed shape of the sample at distance of $500 \mathrm{~mm}$ to the specimen's bottom surface in no mist condition. From this figure, the reconstruction of the outline of the shape can be reproduced. Since the average error of the cylinder height was $0.2 \%$, the measurements in the direction of the ultrasound transmission were accurate. On the other hand, in the radial direction, the error in the evaluation of the circular equivalent was $-16 \%$.

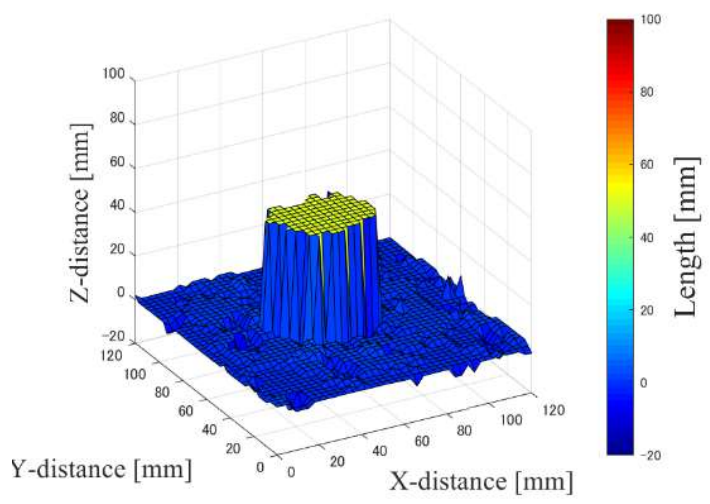

Figure 10: Shape measurement result using parametric sound (Measurement distance $=$ $500 \mathrm{~mm}$, without mist condition). 
Fig. 11 shows the reconstructed shape of the sample at distance of $500 \mathrm{~mm}$ to the specimen's bottom surface in the presence of mist. This figure shows that the reconstruction of the outline can be achieved even in the presence of mist. The error in the height of the cylinder sample was $1.8 \%$ on average, so that the measurements in the direction of the ultrasound transmission were accurate. On the other hand, in the radial direction, the error of the circular equivalent was $-22 \%$, which is considered to be due to the small size of the sample in relation to the width of the ultrasonic beam.

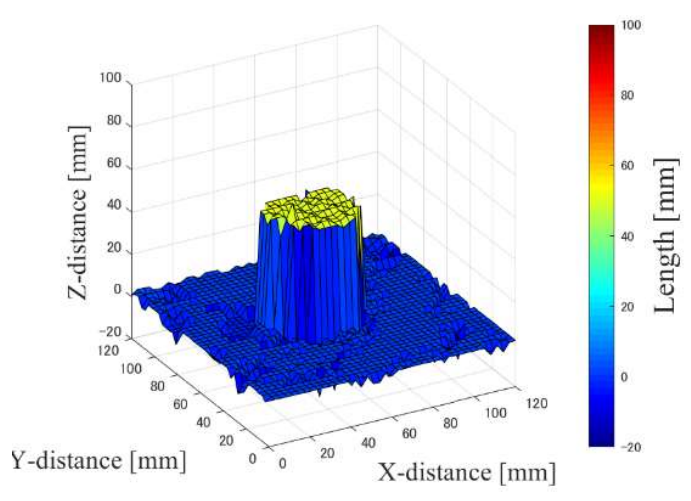

Figure 11: Shape measurement result using parametric sound (Measurement distance $=$ $500 \mathrm{~mm}$, with mist condition).

These results show that parametric sound can be used to measure the shape of the sample even in a mist environment, especially in the ultrasonic transmission direction (Z-axis). On the other hand, for the direction perpendicular to the transmission direction, i.e. XY plane direction, it is guessed that the wide beam width caused errors in the reconstructed shape of the sample. For solving the problem, we need to reduce the size of the transducer and increasing the measurement pitch.

\section{DEVELOPMENT OF SMALL ULTRASONIC MEASUREMENT DEVICE}

To mount an ultrasonic measurement device on the robot arm, we prototyped the ultrasonic transmitter/receiver hardware systems as shown in Figs 12 and 13. The hardware systems consist of a high voltage power supplier, a pulse transmitter, an echo signal amplifier/filter and a micro controller unit (MCU), high voltage pulsar, echo signal amplifier and filter and $\mathrm{A} / \mathrm{D}$ converter. The detailed characterization results of the systems and actual measurement results will be discussed in the presentation.

\section{CONCLUSION}

In order to develop the shape measurement system using parametric sound, we have conducted fundamental experiments to verify the measurable distance and the accuracy of the shape measurement. The measurable distance experiment using parametric sound showed that parametric sound is capable of transmitting and receiving at a distance of 3,500 $\mathrm{mm}$. Shape measurement experiment using parametric sound under no mist or mist conditions showed that it was possible to obtain the outline of the sample shape at a distance of $500 \mathrm{~mm}$. The error in 


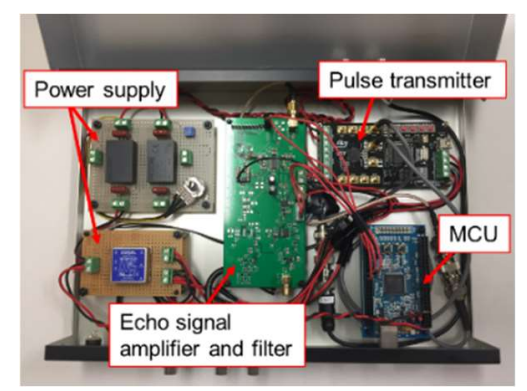

Figure 12: Developed ultrasonic measurement device (UVP-TIT SKP1).

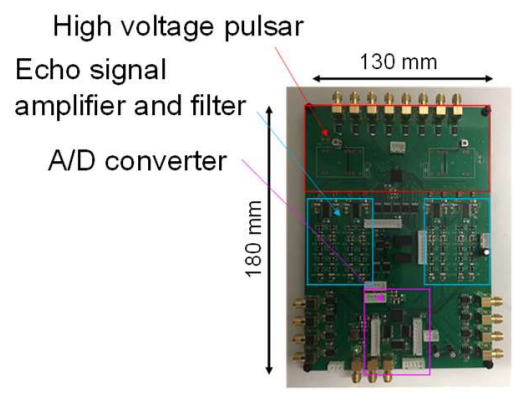

Figure 13: Developed ultrasonic measurement device (UVP-TIT SKP2).

the ultrasonic direction was smaller than the error in the direction perpendicular to the ultrasonic transmission direction in the absence and presence of mist. In addition, we designed and prototyped the ultrasonic measurement device to mount on the robot arm system.

\section{ACKNOWLEDGEMENTS}

This work was partially supported by JAEA Nuclear Energy S\&T and Human Resource Development Project through concentrating wisdom Grant Number JPJA19P 19210348, entitled "Challenge to Investigation of Fuel Debris in RPV by an Advanced Super Dragon Articulated Robot Arm".

\section{REFERENCES}

[1] Tokyo Electric Power Company Holdings, Inc., Mid-and-Long-Term Decommissioning Action Plan 2020. www.tepco.co.jp/en/hd/decommission/information/dap/pdf/ dap_20200327_01-e.pdf. Accessed on: 26 Aug. 2020.

[2] Kikura, H., Takahashi, H., Endo, G. \& Wakaida, I., Fukushima reconstruction support activities and decommissioning research project at Tokyo Institute of Technology "Fukushima revitalizics" and "challenge to investigation of fuel debris in RPV by an advanced Super Dragon articulated robot arm (1)". Presented at Nuclear2020 ASME's Nuclear Engineering Conference powered by ICONE.

[3] Endo G., Takahashi, H. \& Kikura, H., Challenge to investigation of fuel debris in RPV by an advanced Super Dragon articulated robot arm (2) design and prototyping of a lightweight super long reach articulated manipulator. Presented at Nuclear2020 ASME's Nuclear Engineering Conference powered by ICONE. 